\title{
Columnar variant of papillary carcinoma in the thyroglossal duct cyst with progression to lung metastasis
}

\author{
Yujung Yun, Hye Jung Park, Young Ki Lee, Yongin Cho, Beoduel Kang, Hyun Ju Kim, \\ Jung-Hee Lee, Moo-Nyun Jin, Dong Yeob Shin
}

Division of Endocrinology and Metabolism, Department of Internal Medicine, Yonsei University College of Medicine, Seoul, Korea

Thyroglossal duct cyst (TGDC) carcinoma generally shows a favorable prognosis. If metastasis is present latently, it may not threaten the patient's life immediately. It has been shown, however, that larger than $1 \mathrm{~cm}$ papillary carcinoma $(\mathrm{PC})$, level $\mathrm{VI}$ metastasis to the lymph node $(\mathrm{LN})$, which is the nearest to the thyroid, independently predicts a worse prognosis. In the case presented herein, a 61-year-old female patient was diagnosed with an about $3 \mathrm{~cm}$ PC in the TGDC, particularly the columnar variant subtype, one of the aggressive variants. She had occult papillary thyroid microcarcinoma, but no LN metastasis. Even though she underwent the Sistrunk procedure and total thyroidectomy with central compartment neck dissection followed by high-dose radioactive iodine remnant ablation, however, the cancer cells spread to level IV neck LN, and finally to the lung. Therefore, when a patient is diagnosed with an aggressive histologic variant of PC in the TGDC, even without LN metastasis, the invasive surgical approach and close postoperative surveillance are necessary, with consideration of the risk of disease progression. Therefore, if it is possible to stratify the risk for patients, higher-risk patients can be offered a more invasive therapeutic approach.

Keywords: Thyroglossal duct cyst; Papillary carcinoma; Disease management

\section{INTRODUCTION}

A malignancy in the thyroglossal duct cyst (TGDC) is uncommon, with an approximately $1 \%$ occurrence rate of all TGDCs [1]. The standard treatment is surgical resection by means of the Sistrunk procedure, but there is no definite guideline for the surgical extent of thyroid gland and lymph node (LN) dissection when cancer is incidentally found in the TGDC. The invasive surgical treatment for TGDC cancer patients is still controversial because the prognosis is usually favorable. The relative rates of recurrence and distant metastases (less than 2\%) are low, and death related to the disease is uncommon [2]. Based on the specific lymphatic drainage

Received: August 16, 2013, Revised: September 25, 2013, Accepted: September 30, 2013

Corresponding Author: Dong Yeob Shin, Division of Endocrinology and Metabolism, Department of Internal Medicine, Severance Hospital, 50-1 Yonsei-ro, Seodaemun-gu, Seoul 120-752, Korea

Tel: +82-2-2228-5450, Fax: +82-2-362-6835

E-mail: SHINDONGYI@yuhs.ac pathway, however, the cancer cells from a TGDC can primarily spread to the submental (level I) or jugular (levels II-III-IV) LNs without any involvement of the central compartment neck LNs (level VI), which are located farther away from the TGDC. Reported herein is a case of a columnar variant of papillary TGDC carcinoma for which the Sistrunk procedure and total thyroidectomy with central compartment neck dissection (CCND), followed by 131-I remnant ablation, were performed. Lung metastasis was found in the follow-up periods.

\section{CASE}

A 61-year-old female came to Severance Hospital due to a prominent neck mass with anterior neck pain. Physical examination revealed a palpable anterior neck mass without tenderness. In the neck ultrasonography, a $3 \mathrm{~cm}$ hypoechoic mass was found in the pretracheal area, which needed to be examined to determine if its malignancy was evolving from the pyramidal lobe or from the pretracheal lymphadenopathy. A $0.7 \mathrm{~cm}$ nodule suspicious for malignancy was found in the 

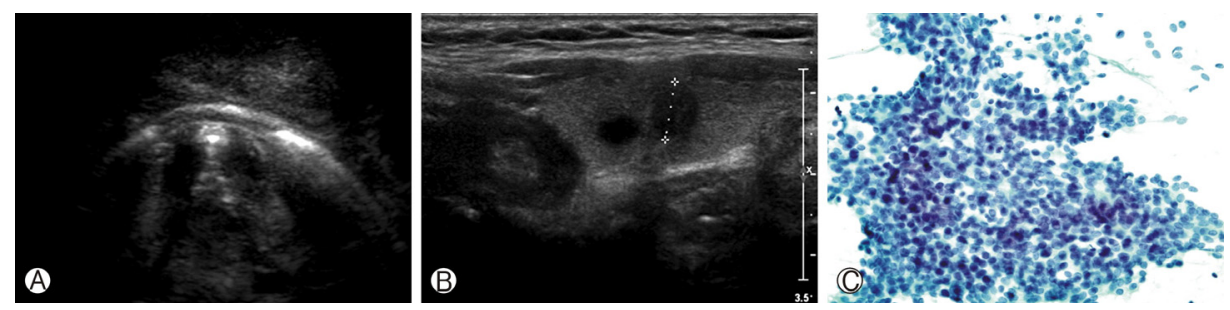

Fig. 1. Large hypoechoic mass (A) and left thyroid nodule (B) in neck ultrasonography. Histopathology (Papanicolaou stain, $\times 200)(C)$.

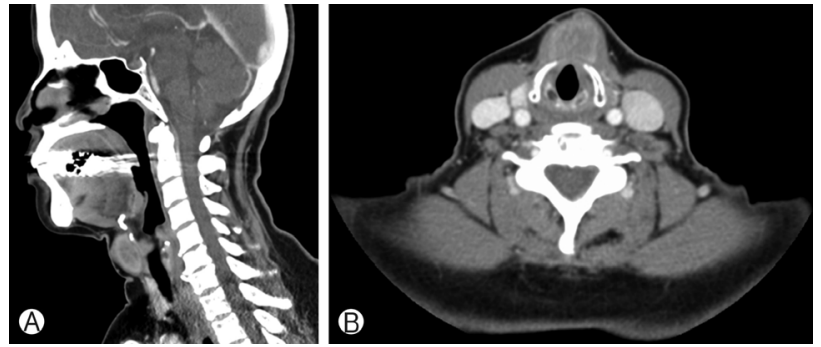

Fig. 2. Thyroglossal duct cyst (TGDC) in computed tomography scan. There was a $2.8 \mathrm{~cm}$ enhancing mass suspicious for malignancy in the TGDC. (A) Sagittal view. (B) Transverse view.

left thyroid lobe (Fig. 1A, 1B). Fine-needle aspiration biopsy (FNAB) was thus performed. The histopathology of the pretracheal mass revealed benign follicular cells, and that of the thyroid nodule in the left lobe revealed papillary thyroid carcinoma (PTC, Bethesda category VI) (Fig. 1C). Computed tomography (CT) scan revealed a $2.8 \mathrm{~cm}$ enhancing mass in the pretracheal area embedded in the strap muscles, which was suspicious for malignancy in a TGDC, and multiple nodules in the left thyroid lobe (Fig. 2). The thyroid function test were within the normal range, and the basal serum thyroglobulin $(\mathrm{Tg})$ level was 157.4 ng/mL (Fig. 3). Fluorodeoxyglucose (FDG) positron emission tomography (PET)-CT demonstrated an about $2 \times 2 \mathrm{~cm}$ enhancing mass in the pretracheal area that showed increased FDG uptake. Both thyroid nodules did not show increased FDG uptake. No evidence of significant cervical LN enlargement was found in all the preoperative imaging studies (Fig. 4).

Total thyroidectomy with CCND and excision of the TGDC were performed (Fig. 5). A $1.5 \times 1.5 \mathrm{~cm}$ slightly hard fixed mass in the left lobe of the thyroid gland was noted. Another mass was a soft and encapsulated cyst, and it continued along the inner margin of the hyoid body. The mass was removed with dissection. The excised cyst was about $2 \times 2 \mathrm{~cm}$ large, including the hyoid body. Postoperative his-

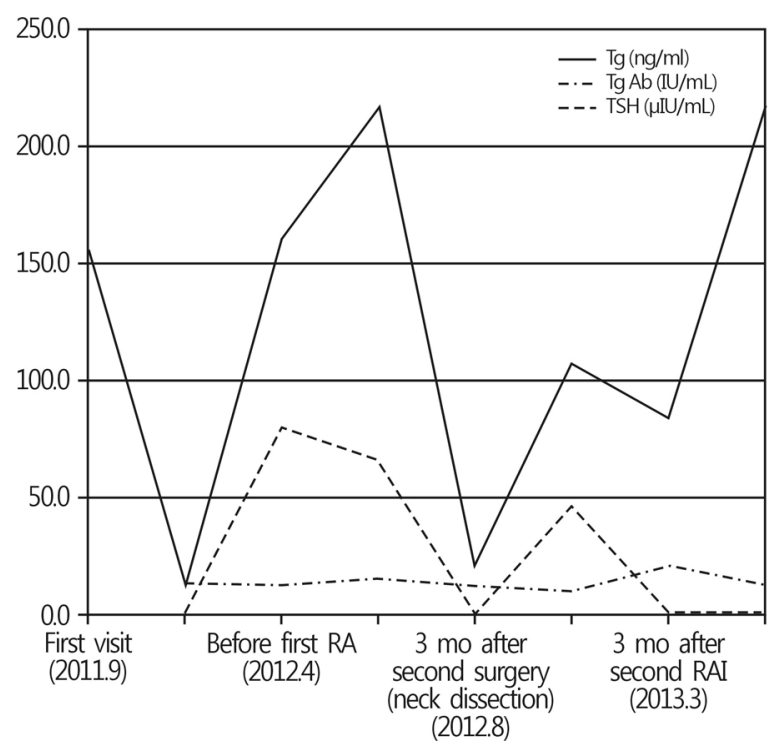

Fig. 3. Changes in the serum markers ( $\mathrm{Tg}, \mathrm{Tg}-\mathrm{Ab}$, and $\mathrm{TSH})$. $\mathrm{Tg}$, thyroglobulin; $\mathrm{Tg}-\mathrm{Ab}$, thyroglobulin-antibody; TSH, thyroid stimulating hormone; RAI, radioactive iodine.
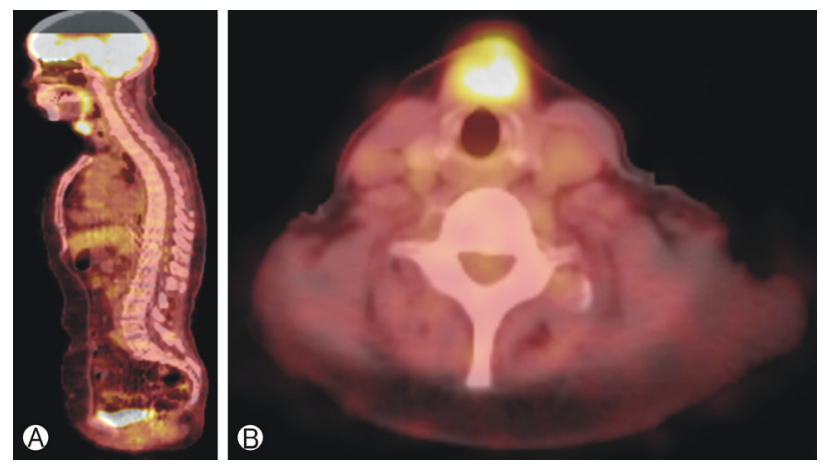

Fig. 4. About $2 \times 2 \mathrm{~cm}$ enhancing mass with increased fluorodeoxyglucose uptake in positron emission tomography-computed tomography. (A) Sagittal view. (B) Transverse view.

topathology revealed a $0.2 \mathrm{~cm}$ diameter infiltrative classical papillary thyroid microcarcinoma (PTMC) at the left thyroid lobe. Psammomatous calcification was absent. It also revealed a $1.8 \mathrm{~cm}$ diameter infiltrative papillary carcinoma (PC) of the 


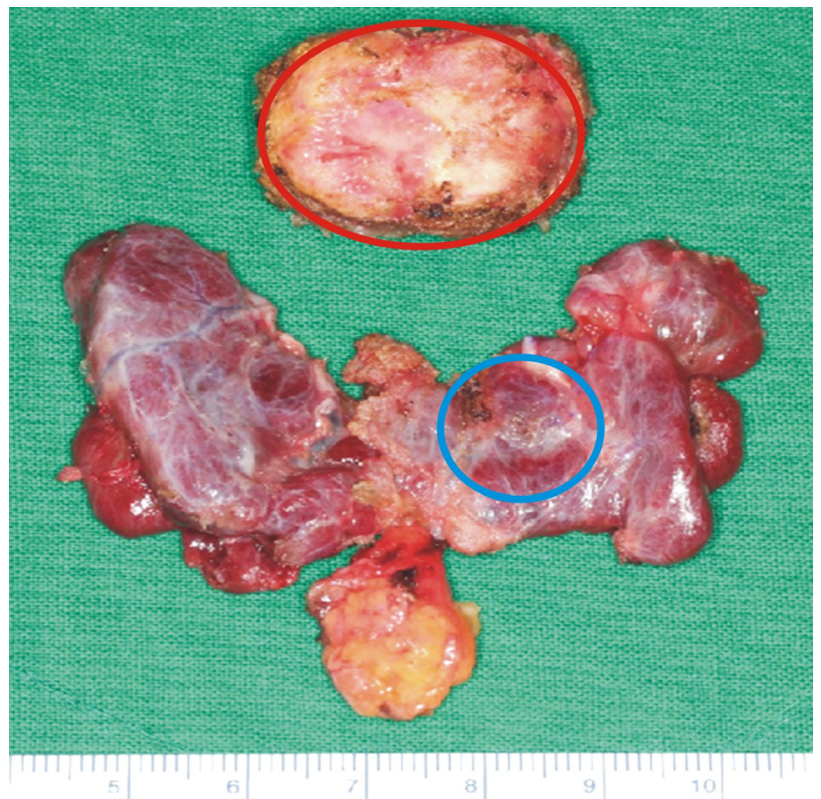

Fig. 5. Thyroglossal duct cyst (upper red circle) and thyroid gland (lower blue circle: the carcinoma was located).

TGDC that extended to the adjacent skeletal muscle. The histologic subtype was predominantly a columnar cell variant and partly the conventional type. Psammomatous calcification was present and subnuclear and supranuclear vacuoles were seen (Fig. 6). The intrathyroidal mass of the PTC showed no microscopic extrathyroidal extension, and there was no $\mathrm{LN}$ metastasis. The level of postoperative serum $\mathrm{Tg}$ was 10.6 $\mathrm{ng} / \mathrm{mL}$, within the normal range $(0-39.2 \mathrm{ng} / \mathrm{mL})$ (Fig. 3). The patient underwent a postoperative high dose $(150 \mathrm{mCi})$ of radioiodine remnant ablation (RRA) 6 months after the surgery. The post-radioiodine whole-body scan showed multiple foci of increased radioiodine uptake in the neck, probably remnant thyroid tissue. The stimulated Tg level was 158.6 $\mathrm{ng} / \mathrm{mL}$ when the thyroid-stimulating hormone (TSH) level was $79.84 \mu \mathrm{IU} / \mathrm{mL}$ (Fig. 3). A follow-up PET-CT that was performed simultaneously with RRA showed right-neck level IV LN enlargement with increased FDG uptake, which may suggest cancer metastasis (Fig. 7). Another right-neck level II enlarged LN with an oval shape was possibly benign. Neck ultrasonography at the same time showed an approximately $1.0 \times 0.6 \times 1.5 \mathrm{~cm}$ right-neck level IV enlarged LN that also suggests $\mathrm{LN}$ metastasis. There was no evidence of tumor recurrence at the operation site (Fig. 8). FNAB was performed for the suspicious metastatic lateral neck LN. The cytological result was positive for malignancy (metastatic PC), and the
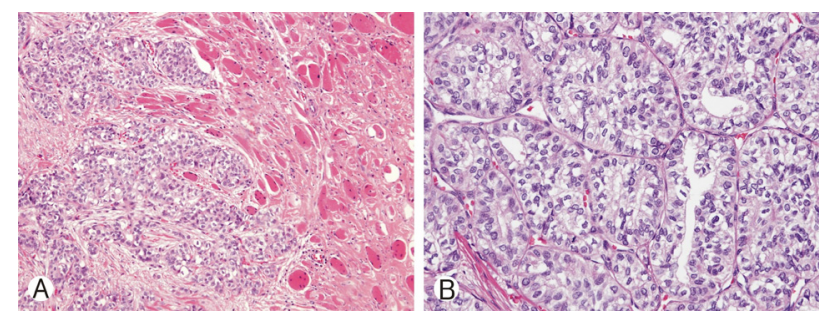

Fig. 6. Postoperative histopathology. There was a $1.8 \mathrm{~cm}$ diameter papillary carcinoma with a predominantly columnar cell variant in the thyroglossal duct cyst. (A) The tumor is invading adjacent skeletal muscle (H\&E stain, $\times 100)$. (B) The comlumnar cell variant is present and subnuclear and supranuclear vaculoes are seen (H\&E stain, $\times 200)$.
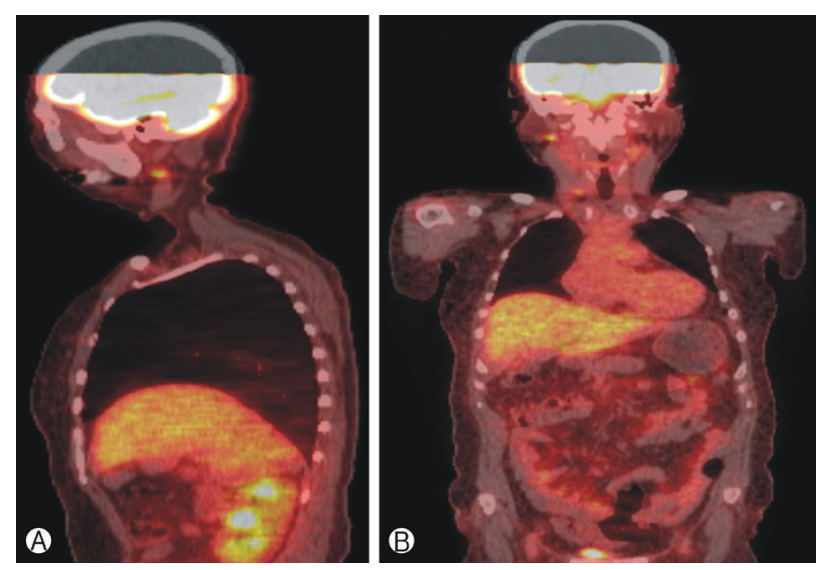

Fig. 7. Right-neck level IV lymph node enlargement with fluorodeoxyglucose uptake in the follow-up positron emission tomography-computed tomography after the first radioiodine remnant ablation. (A) Sagittal view. (B) Coronal view.

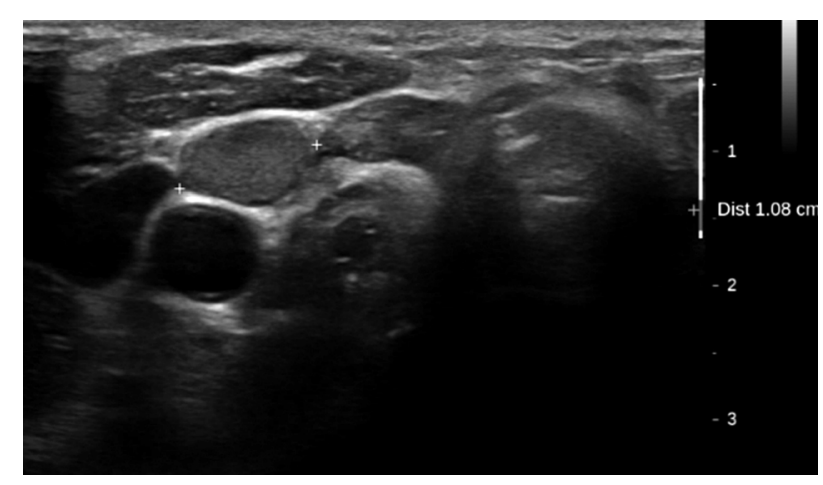

Fig. 8. Right-neck level IV lymph node enlargement in neck ultrasonography.

level of wash-out $\mathrm{Tg}$ was above $1,000 \mathrm{ng} / \mathrm{mL}$. The serum $\mathrm{Tg}$ level was up to $217 \mathrm{ng} / \mathrm{mL}$ (Fig. 3). Neck magnetic resonance imaging also revealed right-neck level IV enlarged LNs, 

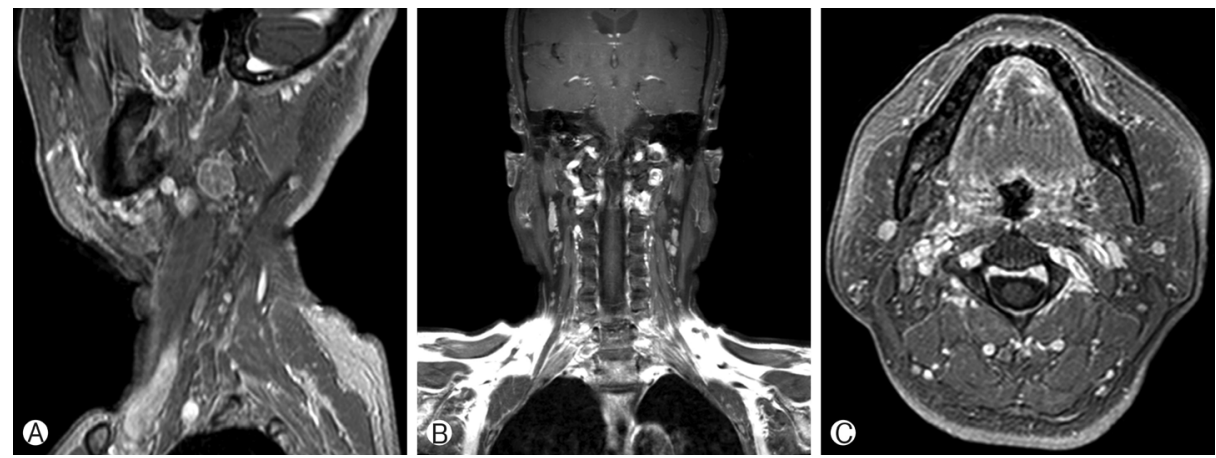

Fig. 9. Right-neck level IV lymph node (LN) enlargement in neck magnetic resonance imaging. It suggests metastatic LNs. (A) Sagittal view. (B) Coronal view. (C) Transverse view.

suggesting metastatic LNs (Fig. 9). After 7 weeks from the first postoperative RRA, the patient underwent right selective neck dissection at levels IIa, IIb, III, IV and V and the postoperative pathology revealed metastatic carcinoma in one (at level III) of the $25 \mathrm{LNs}$, without perinodal soft-tissue extension. Afterwards, the second radioactive iodine (150 $\mathrm{mCi})$ treatment was performed, and in the postradioiodine whole-body scan, there was no definite focus of increased radioiodine uptake noted in the neck area. The stimulated $\mathrm{Tg}$ level assessed immediately before radioiodine administration was $106.5 \mathrm{ng} / \mathrm{mL}$, when TSH was $47.06 \mu \mathrm{IU} / \mathrm{mL}$ and the $\mathrm{Tg}$ antibody level was $10.9 \mathrm{IU} / \mathrm{mL}$ (Fig. 3). The FDG-PET whole-body scan performed simultaneously with the second radioiodine treatment showed no discernible FDG uptake suggesting tumor recurrence.

Three months after the second radioiodine treatment, the basal Tg level was assessed to be $83.4 \mathrm{ng} / \mathrm{mL}$ when the level of serum $\mathrm{Tg}$ antibody was $19.9 \mathrm{IU} / \mathrm{mL}$ (normal range: 0-130.6), and a serum TSH was undetectable $(<0.025 \mathrm{IU} / \mathrm{mL}$; normal range, 0.35-4.94) (Fig. 3). At the same time, neck ultrasonography revealed the presence of a small (less than $5 \mathrm{~mm}$ ) LN on the left neck area (level IV). Then the patient was followed up after three months with FNAB and non-contrast chest CT scan. The FNAB cytology result turned out to be negative for malignancy and the wash-out $\mathrm{Tg}$ level was low $(3.9 \mathrm{ng} / \mathrm{mL})$. The basal serum $\mathrm{Tg}$ level, however, went up to $216 \mathrm{ng} / \mathrm{mL}$ (Fig. 3 ), and the chest CT scan demonstrated multiple small lung nodules mainly in the lower lobes, suggesting hematogenous lung metastasis (Fig. 10). The patient refused lung biopsy, and she is currently planning to receive the third radioiodine treatment with a higher dose $(200 \mathrm{mCi})$.
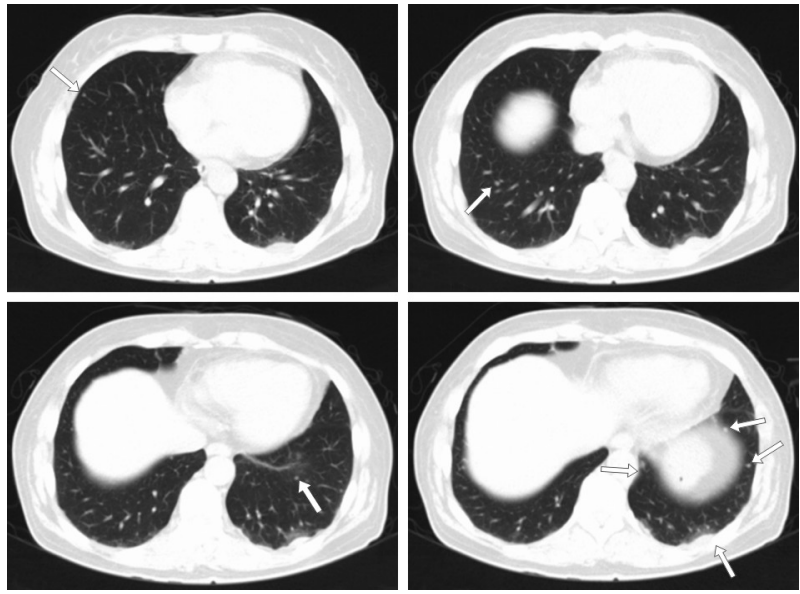

Fig. 10. Ruled-out hematogenous lung metastasis in chest computed tomography scan. There were multiple small lung nodules (arrows indicate) mainly in the lower lobes, suggesting hematogenous lung metastasis.

\section{DISCUSSION}

In the preoperative evaluation of TGDC carcinomas with concomitant thyroid disease, ultrasonography and FNAB are reliable tools in clinical practice. At least $85 \%$ of TGDC carcinomas have been diagnosed as PCs [3], while follicular and anaplastic carcinomas are very rare (both 1\%). PCs have a favorable prognosis irrespective of whether they occur in a TGDC or in a thyroid gland. The occurrence rate of $\mathrm{LN}$ metastasis is lower in PTC arising from a TGDC than in that arising from a thyroid gland [3]. The FNAB of a TGDC generally shows mature squamous cells and inflammatory cells with mucoid materials and a proteinaceous background. Columnar epithelial cells may also be present [4]. PTC has 
various pathological subtypes, including follicular, encapsulated, diffuse sclerosing and columnar and tall cell variants. The columnar and tall cell variants of PTC are associated with more aggressive progression and a lower survival rate compared to the other variants of PTC [5,6]. Köybaşioğlu et al. have reported a patient with a tall-cell-variant PC arising from a TGDC, showing a poor prognosis. It has been reported that the incidence of tall-cell-variant PTC is about 9\% of all PTCs. It tends to develop in older patients and to be bigger than $5 \mathrm{~cm}$. Johnson et al. found $75 \%$ involvement of the cervical LNs by the tumor, a $42 \%$ extrathyroidal extension rate, a $58 \%$ local recurrence rate, and a $17 \%$ incidence of distant metastases [6]. It is known that the columnar cell variants of PC arising from a TGDC also have poor prognosis, but nearly no case of a patient with a columnar variant has been reported. It has to be discussed what should be considered in selecting the therapeutic approach of a patient with an aggressive variant of TGDC, which is predicted to have a poor prognosis, as in the case reported herein.

In 1920, Sistrunk discovered a method of performing surgery for the TGDC considering its embryologic origin. This surgery included resection of the cyst and the tract, which extends to the foramen cecum at the base of the tongue, in continuity with the mid-portion of the hyoid bone [7]. Until now, there have been long discussions about the method and extent of the surgical resection.

In previous reports, a synchronous intrathyroidal pathology has been reported in up to $30 \%$ of TGDC carcinomas [8]. Synchronous malignancy in the thyroid may represent 2 independent primary carcinomas, metastasis from a primary carcinoma in the cyst, or a PTC with metastatic involvement of the TGDC. If there is such a suspicious lesion in the thyroid gland of a patient with TGDC carcinoma, total thyroidectomy should also be performed. Such total thyroidectomy combined with Sistrunk surgery decreases the recurrence rate and makes postoperative follow-up easier by monitoring the serum $\mathrm{Tg}$ level [9].

The strategic method of $\mathrm{LN}$ dissection is also controversial. Cervical LN metastasis from the PC of the TGDC has been reported in $7 \%$ to $15 \%$ of the cases [1]. In the case of a welldifferentiated thyroid carcinoma with no LN metastasis, selective cervical LN dissection for TGDC may be unnecessary, but when the cervical lymphadenopathy is suspicious, therapeutic management including the Sistrunk procedure, total thyroidectomy, and appropriate-level selective neck dissection followed by postoperative adjuvant radioiodine therapy is recommended. Dzodic et al. placed particular emphasis on the importance of central compartment neck (level VI) dissection for TGDC patients with thyroid lesions suspected of being carcinomas [10]. They performed prophylactic level VI dissections for the patients with nodules suspicious for thyroid carcinoma because re-operation for the central compartment neck LN recurrence can cause severe complications, such as recurrent laryngeal nerve injury and persistent hypoparathyroidism. They likewise found that the patients with thyroidal PTC lesions also had metastases in the central neck compartment LN. Hartl et al. reported that a PC arising in a TGDC has occult LN metastases especially in the neck (86\%). They suggested that "skip" metastases to the lateral neck seem to be more frequent than for PC arising in the thyroid lobes (40\% in this study as compared to only $20 \%$ in usual cases of papillary thyroid cancer). Therefore, it should be kept in mind that lateral neck dissection may be needed secondarily in midline tumors [11].

Patel et al. suggested that patients with TGDC carcinoma in the "higher-risk group" classified based on criteria including older than 45 years old, larger than $4 \mathrm{~cm}$ tumors, or soft-tissue extension with nodal or distant metastases require more aggressive management encompassing the Sistrunk procedure and total thyroidectomy with or without neck dissection, followed by radioidine therapy [8].

It is known that PCs have a prolonged clinical course and a low mortality rate, requiring long-term follow-up of the patients ( $>20$ years). High concentrations of FNAB washout $\mathrm{Tg}$ in a cystic cervical mass determined via radiological imaging study are considered specific to PTC LN metastasis, indicating their usefulness in distinguishing PTC cystic metastasis from other cystic lesions. Wash-out Tg measurement with FNAB may thus improve the preoperative diagnosis accuracy without additionally stressing the subjects with PTC cystic LN metastasis [12].

The existing guidelines regarding the management of patients with TGDC carcinomas are insufficient because no large studies and long-term follow-up have been done. In the case reported herein, when the patient was diagnosed with PC in TGDC with occult PTMC, she had no LN metastasis, but even though she underwent surgery and postoperative high-dose radioactive iodine, the cancer cells spread to the lung. Therefore, when a patient is diagnosed with the columnar variant, one of the 
aggressive variants of PC in TGDC, even though there is no $\mathrm{LN}$ metastasis, the invasive surgical approach and close postoperative surveillance are definitely necessary due to the risk of disease progression, as in the case reported herein.

\section{ACKNOWLEDGEMENT}

This work was supported by the professors of endocrinology at Severance Hospital.

\section{REFERENCES}

1. Motamed M, McGlashan JA. Thyroglossal duct carcinoma. Curr Opin Otolaryngol Head Neck Surg 2004;12:106-9.

2. Pellegriti G, Lumera G, Malandrino P, Latina A, Masucci R, Scollo C, et al. Thyroid cancer in thyroglossal duct cysts requires a specific approach due to its unpredictable extension. J Clin Endocrinol Metab 2013;98:458-65.

3. Yang YJ, Haghir S, Wanamaker JR, Powers CN. Diagnosis of papillary carcinoma in a thyroglossal duct cyst by fine-needle aspiration biopsy. Arch Pathol Lab Med 2000;124:139-42.

4. Astl J, Dusková J, Kraus J, Vlcek P, Kodet R, Lastůvka P, et al. Coincidence of thyroid tumor and thyroglossal duct remnants. review of the literature and presentation of three cases. Tumori 2003;89:314-20.

5. Hari CK, Brown MJ, Thompson I. Tall cell variant of papillary carcinoma arising from ectopic thyroid tissue in the trachea. J Laryngol Otol 1999;113:183-5.

6. Köybaşioğlu F, Simşek GG, Onal BU. Tall cell variant of papillary carcinoma arising from a thyroglossal cyst: report of a case with diagnosis by fine needle aspiration cytology. Acta Cytol 2006;50:221-4.

7. Sistrunk WE. The surgical treatment of cysts of the thyroglossal tract. Ann Surg 1920;71:121-2.

8. Patel SG, Escrig M, Shaha AR, Singh B, Shah JP. Management of well-differentiated thyroid carcinoma presenting within a thyroglossal duct cyst. J Surg Oncol 2002;79:134-9.

9. Miccoli P, Minuto MN, Galleri D, Puccini M, Berti P. Extent of surgery in thyroglossal duct carcinoma: reflections on a series of eighteen cases. Thyroid 2004;14:121-3.

10. Dzodic R, Markovic I, Stanojevic B, Saenko V, Buta M, Djurisic I, et al. Surgical management of primary thyroid carcinoma arising in thyroglossal duct cyst: an experience of a single institution in Serbia. Endocr J 2012;59:517-22.

11. Hartl DM, Al Ghuzlan A, Chami L, Leboulleux S, Schlumberger M, Travagli JP. High rate of multifocality and occult lymph node metastases in papillary thyroid carcinoma arising in thyroglossal duct cysts. Ann Surg Oncol 2009;16:2595-601.

12. Inagaki Y, Sakamoto K, Inoue Y, Imanishi Y, Tomita T, Shinden S, et al. [Thyroglobulin concentration measurement in fine-needle aspiration fluid from cystic cervical lymph node metastases of papillary thyroid carcinoma]. Nihon Jibiinkoka Gakkai Kaiho 2011;114:912-6. 